\title{
Effects of quantum noise on the nonlinear dynamics of a semiconductor laser subject to two spectrally filtered, time-delayed optical feedbacks
}

\author{
Joseph S. Suelzer ${ }^{\mathrm{a}, *}$, Awadhesh Prasad ${ }^{\mathrm{b}}$, Rupamanjari Ghosh $^{\mathrm{c}}$, Gautam Vemuri ${ }^{\mathrm{a}}$ \\ ${ }^{a}$ Department of Physics, Indiana University Purdue University Indianapolis (IUPUI) Indianapolis, IN 46202-3273 \\ ${ }^{b}$ Department of Physics and Astronomy, University of Delhi, Delhi 110007, India \\ ${ }^{c}$ Shiv Nadar University, NH-91, Tehsil Dadri, Gautam Buddha Nagar, UP 201314, India
}

\begin{abstract}
We report on a theoretical and computational investigation of the complex dynamics that arise in a semiconductor laser that is subject to two external, time-delayed, filtered optical feedbacks with special attention to the effect of quantum noise. In particular, we focus on the dynamics of the instantaneous optical frequency (wavelength) and its behavior for a wide range of feedback strengths and filter parameters. In the case of two intermediate filter bandwidths, the most significant results are that in the presence of noise, the feedback strengths required for the onset of chaos in a period doubling route are higher than in the absence of noise. We find that the inclusion of noise changes the dominant frequency of the wavelength oscillations, and that certain attractors do not survive in the presence of noise for a range of filter parameters. The results are interpreted by use of a combination of phase portraits, rf spectra, and first return maps.
\end{abstract}

Keywords: elsarticle.cls, $\mathrm{HT}_{\mathrm{E}} \mathrm{X}$, Elsevier, template

2010 MSC: 00-01, 99-00

\section{Introduction}

A semiconductor laser (SCL) subject to feedback has become a paradigm for studying nonlinear dynamics in time-delayed feedback systems. At a fundamental level, 5 studies on such systems are an ideal test bed for delay systems, and at an applied level, some of the dynamical behaviors have been exploited for cryptography, random-bit generation and even understanding of collective neuronal excitations in the brain [1, 2, (see references therein)]. $\mathrm{A}^{35}$ 10 number of impressive studies on electronic feedback and all-optical feedback, and the resulting dynamics in a SCL have been reported over the years 3 , 4. Within the context of all-optical feedback, investigators have studied conventional optical feedback where a mirror is placed in front

15 of the SCL such that a fraction of the light from the laser is reflected back into it [5, 6]. Other feedback scenarios have included polarization rotated feedback where the polarization of the feedback light is rotated relative to the dominant polarization mode of the laser light $7,8,8,9$. Re- ${ }^{45}$ 20 cently, the effects of injection on the stability properties from two polarization modes has been studied [10.

Another interesting feedback scheme that has been reported is filtered optical feedback (FOF) wherein the feedback light is spectrally filtered before entering the laser ${ }^{50}$

25 11. FOF provides the user with two additional parameters, the bandwidth of the filter and the detuning between

\footnotetext{
* Corresponding author

Email address: josesuel@iupui.edu (Joseph S. Suelzer)
}

the filter frequency and the laser light frequency, to control the dynamics of the laser. One of the major dynamical effects observed in FOF is that the frequency of the laser light, for a judicious choice of filter parameters, exhibits controlled oscillations at a frequency that is related to the time delay of the feedback 12. It has also been shown that for other filter parameters, one can observe a period doubling route to chaos in the frequency of the laser light [13.

Recently, there has been an interest in the dynamics of a SCL subject to two simultaneous FOFs 14, 15. Krauskopf and co-workers have reported an exhaustive study of the bifurcations that arise in such systems [16, 17. The use of two filters provides a number of additional parameters that can be potentially used to control the dynamics of the SCL. Our group reported an experimental study on the frequency dynamics in the light from the laser when subject to two FOFs [18]. Among the more interesting observations was the generation of new frequencies in the system, and the results were explained via a theoretical model that consisted of the usual Lang-Kobayashi rate equations augmented to include two FOFs. The agreement between experiments and theory was excellent.

One of the observations that emerged from our prior work was that the frequency of laser light in a SCL subject to two FOFs follows a period-doubling route to chaos. However, the feedback strength necessary for coherence collapse that was predicted by the theoretical model was 55 higher than what was observed experimentally. This mis- 


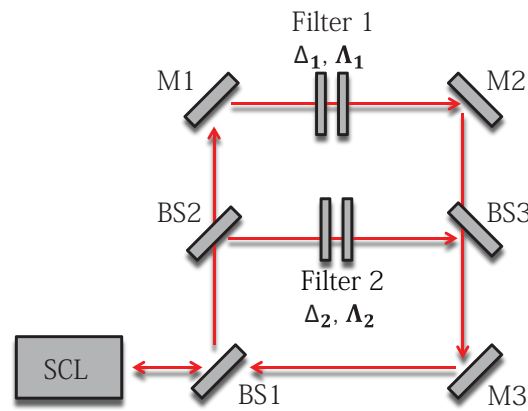

Figure 1: The schematic shows a semiconductor laser (SCL) subject 80 to optical feedback from two external cavities. The outer cavity (1) is formed by the SCL and the mirrors (M1, M2, and M3). The inner cavity (2) is formed by the SCL, beam splitters (BS2 and BS3), and mirror (M3). Each cavity contains a Fabry-Perot resonator acting as a spectral filter, which can be modified by changing the reflectivity or spacing of the filter mirrors. This in turn changes the bandwidth, $\Lambda$. The detuning, $\Delta$, is altered by adjusting the pump current. The delay-times, $\tau_{1}$ and $\tau_{2}$, are increased or decreased by lengthening or shortening the cavities.

match between theory and experiment inspired us to examine the role of unavoidable quantum noise in the laser and its influence on the dynamics of the laser. To this end, we have augmented the theoretical model with Langevin noise terms to account for the spontaneous emission noise as well as inversion noise. To give one a picture of the system studied, we show a schematic of the experimental design [Fig,1] highlighting the key parameters accessible to experiment, which are detailed in the following section.

\section{Model}

A semiconductor laser with FOF from a single cavity can be modeled with a set of rate equations describing the time evolution of the slowly varying complex electric fields, $E(t)$ and $F(t)$, of the laser and feedback field, respectively, and the carrier inversion $N(t)$ [1]. Our setup [Fig.11, which includes two cavities, each with a spectral filter, 85 must therefore include two filtered feedback fields, $F_{1}(t)$ and $F_{2}(t)$, resulting in the following description,

$$
\frac{d E}{d t}=\frac{1}{2}(1+i \alpha) \xi N(t) E(t)+\kappa_{1} F_{1}\left(t, \tau_{1}\right)+\kappa_{2} F_{2}\left(t, \tau_{2}\right)+L_{E}(t)
$$

$\frac{d N}{d t}=J-J_{t h r}-\frac{N(t)}{T_{1}}-\left[\Gamma_{0}+\xi N(t)\right]|E(t)|^{2}+L_{N}(t)$,

$\frac{d F_{1}}{d t}=\Lambda_{1} E\left(t-\tau_{1}\right) \mathrm{e}^{-i \omega_{0} \tau_{1}}+\left(i \Delta_{1}-\Lambda_{1}\right) F_{1}(t)$.

$\frac{d F_{2}}{d t}=\Lambda_{2} E\left(t-\tau_{2}\right) \mathrm{e}^{-i \omega_{0} \tau_{2}}+\left(i \Delta_{2}-\Lambda_{2}\right) F_{2}(t)$,

where $\xi N E$ in the first term of Eq. (1a) accounts for the100 growth (or decay) when the carrier inversion $N(t)$ is above (or below) threshold, and $\xi$ is the differential gain coefficient. $\alpha$ is the linewidth enhancement factor which quantifies the phase-amplitude coupling. The second (third) term in Eq. (1a) accounts for the feedback field $F_{1}\left(t, \tau_{1}\right)$ $\left(F_{2}\left(t, \tau_{2}\right)\right)$, where $\kappa_{1}\left(\kappa_{2}\right)$ is the feedback rate and $\tau_{1}\left(\tau_{2}\right)$ is the time delay due to the propagation of the feedback 75 field in cavity 1 (2).

The rate equation for the carrier inversion [Eq. (1b)] depends on the difference between the pump current $J$ and the threshold current $J_{t h r}$. The second term [Eq. (1b]] accounts for the spontaneous decay, hence $T_{1}$ is the carrier decay rate, and the third term includes the stimulated emission, where $\Gamma_{0}$ is the photon decay rate.

Eqs. (1c) and (1d) are derived by assuming that the response function, $r(\omega)$, of the filter is Lorentzian [11, i.e.

$$
r(\omega)=\frac{\Lambda}{\Lambda+i(\omega-\Delta)},
$$

where $\omega$ is the instantaneous optical frequency, $\Lambda$ is the half width at half maximum (HWHM) of the filter and $\Delta$ is the detuning of the solitary laser frequency from the center frequency of the filter. The explicit dependence on the time-delayed fields $E\left(t-\tau_{1,2}\right)$ is seen in Eqs. (1c) and (1d). The feedback phase accumulated due to the propagation of the field through cavity 1 (2) is given by $\omega_{0} \tau_{1}$ $\left(\omega_{0} \tau_{2}\right)$, which we assume equal for the two cavities and fix at $\omega_{0} \tau_{1}=\omega_{0} \tau_{2} \equiv \theta$. The spontaneous-recombination noise terms are described by $L_{E}(t)$ and $L_{N}(t)$. Both noise sources are assumed to be Gaussian with zero mean. Their autocorrelation functions are given by,

$$
\left\langle\operatorname{Re}\left(L_{E}(t)\right) \operatorname{Im}\left(L_{E}\left(t^{\prime}\right)\right)\right\rangle=0,
$$

$$
\left\langle\operatorname{Re}\left(L_{E}(t)\right) \operatorname{Re}\left(L_{E}\left(t^{\prime}\right)\right)\right\rangle=\left\langle\operatorname{Im}\left(L_{E}(t)\right) \operatorname{Im}\left(L_{E}\left(t^{\prime}\right)\right)\right\rangle=R_{s p} \delta\left(t-t^{\prime}\right),
$$

$$
\left\langle L_{N}(t) L_{N}\left(t^{\prime}\right)\right\rangle=D \delta\left(t-t^{\prime}\right) \text {. }
$$

The rate of spontaneous emission is written as $R_{s p}=$ $\beta_{s p} C N^{2}$, where $\beta_{s p}$ is the fraction of spontaneous emission coupled into the dominant mode and $\mathrm{C}$ is a radiative recombination rate 19 . The shot noise diffusion rate is given by $D$. The noise sources $L_{E}$ and $L_{N}$ are derived quantum mechanically in order to arrive at their explicit form and statistical properties [20. Although the noise sources are correlated in a SCL 20, we found that correlating sources resulted in no significant difference when compared to uncorrelated noise [21]. Therefore, the proceeding analysis is done with uncorrelated noise.

Eqs. (1) are integrated using a modified fourth order Runge-Kutta method. The time step was varied from 0.1 ps to $10 \mathrm{ps}$ in order to ensure consistent dynamical behavior independent of the integration step size. The modified Runge-Kutta method accounts for the Langevin noise source and avoids an infinite variance of the stochastic terms following the stochastic Runge-Kutta algorithm outlined in Ref. 22]. At each integration step, the noise terms 
are pulled from a matrix of normally distributed random 135 numbers generated via the $\operatorname{randn}($ ) function in MATLAB. In order to account for the time delayed fields, a history function is built by initially integrating Eqs. (1) without feedback $\left(\kappa_{1,2}=0\right)$. The transient behavior is discarded and the history function with a minimum integration time ${ }_{140}$ of $4 \tau_{1,2}$ is saved. The remaining parameters and noise strengths for a typical edge emitting SCL are highlighted in Table 1 .

Table 1: The parameter values for a typical SCL which are used in ${ }^{145}$ the simulations (unless otherwise specified).

\begin{tabular}{lll}
\hline Quantity & Symbol & Value \\
\hline Linewidth enhancement factor & $\alpha$ & 5 \\
Feedback rate field 1 & $\kappa_{1}$ & Varies \\
Feedback rate field 2 & $\kappa_{2}$ & $0.8 \mathrm{GHz}$ \\
Bandwidth of filter 1 & $\Lambda_{1}$ & Varies \\
Detuning of filter 1 & $\Delta_{1}$ & Varies \\
Bandwidth of filter 2 & $\Lambda_{2}$ & $1.0 \mathrm{GHz}$ \\
Detuning of filter 2 & $\Delta_{2}$ & $-1.5 \mathrm{GHz}$ \\
Delay-time field 1 & $\tau_{1}$ & $14.28 \mathrm{~ns}$ \\
Delay-time field 2 & $\tau_{2}$ & $7.93 \mathrm{~ns}$ \\
Phase accumulation & $\theta$ & 1.111 \\
Differential gain coefficient & $\xi$ & $5 \times 10^{3} \mathrm{~s}^{-1}$ \\
Photon decay rate & $\Gamma_{0}$ & $10^{11} \mathrm{~s}^{-1}$ \\
Carrier decay rate & $T_{1}$ & $1 \mathrm{~ns}$ \\
Threshold pump rate & $J_{t h r}$ & $1 \times 10^{17} \mathrm{~s}^{-1}$ \\
Pump rate & $J$ & $1.5 J_{t h r}$ \\
Spontaneous emission rate & $R_{s p}$ & $5 \times 10^{12} \mathrm{~s}^{-1}$ \\
Shot noise diffusion rate & $D$ & $1.45 \times 10^{16} \mathrm{~s}^{-1}$ \\
\hline
\end{tabular}

We point out that this system contains a rich variety of parameters that are accessible in an experiment. Adjusting the pump current $J$ controls the optical frequency, which in turn changes the detuning $\Delta_{1,2}$. Changing the filter mirror spacing or reflectivity modifies the bandwidth ${ }^{170}$ $\Lambda_{1,2}$, and the time delays $\tau_{1,2}$ are directly proportional to the external cavity lengths $L_{1,2}$. The feedback rates $\kappa_{1,2}$ are controlled via a neutral density filter inserted in each cavity.

\section{Results: a period doubling route to chaos}

This section describes the results of our work, but before doing so it is important to point out that the $2 \mathrm{FOF}_{180}$ system has a large set of parameters, each of which can influence the resulting dynamics. We have, therefore, focused on a limited set which highlights the role of quantum noise. For example, we have chosen typical noise strengths and have not varied the strength of the noise. Furthermore, we have fixed the bandwidth of one of the filters to $1 \mathrm{GHz}$, the so-called intermediate bandwidth which lies ${ }^{185}$ between the external cavity mode spacing frequency and the laser relaxation oscillation (RO) frequency, and varied the bandwidth of the second filter to study the effects of filter bandwidth.
We begin by describing the results of our calculations, shown in Fig. 2, which exhibits a period doubling route to chaos of the frequency $\omega(t)$ of the laser wavelength (instantaneous frequency) oscillations. The period doubling route is produced via the bifurcation parameter $\kappa_{1}$, which is the feedback rate of field 1 . The color scheme corresponds to the amplitude of the oscillations. All other parameters are fixed as specified in Table 1 except the filter bandwidth and detuning, where $\Lambda_{1}=1 \mathrm{GHz}$ and $\Delta_{1}=-0.5 \mathrm{GHz}$. Initially the feedback is solely from cavity $2\left(\kappa_{2}=0.8 \mathrm{GHz}\right.$ and $\left.\kappa_{1}=0 \mathrm{GHz}\right)$. We calculate the time series for $E(t), F_{1,2}(t)$, and $N(t)$. Using the complex electric field $E(t)$ we extract the phase $\phi(t)$, where $\phi(t)=\arg (E(t)) . \arg (z)$ is the typically defined complex argument of $z$. The frequency $\omega(t)$ of the slowly varying complex electric field $E(t)$ is found by calculating the time derivative of the phase $[\omega(t)=\dot{\phi}(t)]$. Finally, the numerical spectra are determined for $E(t)$ and $\omega(t)$ after discarding the transient behavior. The feedback rate $\kappa_{1}$ is increased by $\Delta \kappa_{1} \approx 0.32 \mathrm{GHz}$ and this process is repeated over 32 iterations in order to arrive at a final feedback rate of $\kappa_{1}=10 \mathrm{GHz}$. In the presence of noise, this procedure is averaged over 1000 instances. These spectra are stitched together resulting in a period doubling route to chaos shown in Fig.2 2 Examining the deterministic period doubling map [Fig. 23], the first frequency (labeled $\mathrm{f}_{\mathrm{I}}$ ) to emerge is $105 \mathrm{MHz}$ corresponding to the fundamental frequency from cavity $2\left(f_{2} \approx \frac{1}{\tau_{2}+1 / \Lambda_{2}} \approx 107 \mathrm{MHz}\right)$. Note that the fundamental frequency, which we label as $f_{f u n}$, for FOF is dependent on both the delay time $\tau$ and the bandwidth $\Lambda$. $\kappa_{1}$ is increased and a frequency $\left(\mathrm{f}_{\mathrm{II}}\right)$ of 130 $\mathrm{MHz}$ is produced which corresponds to an average between the fundamental frequency of cavity 2 and the second harmonic of cavity $1\left(\frac{f_{2}+2 f_{1}}{2} \approx 125 \mathrm{MHz}\right)$. A further increase in $\kappa_{1}$ results in a quasi period-doubling route to chaos. Examining the deterministic case in Fig. 2a, it is clear that the onset of chaos begins at $\kappa_{1} \approx 4.5 \mathrm{GHz}$. When noise is present, shown in Fig. 2p, the period doubling route for smaller frequencies is drastically altered. The onset of chaos is delayed and a spread in the spectrum does not emerge until $\kappa_{1} \approx 7 \mathrm{GHz}$. Not only is the chaotic regime shifted to a larger feedback rate, but the frequency content is altered. The stochastic spectra, which are extended to $5 \mathrm{GHz}$ and shown in Fig.2. $\kappa_{1} \approx 4.5 \mathrm{GHz}$. The deterministic spectra (not shown) are very similar when depicted on the same scale. One gains an insight into the mechanism for this delay by examining the dynamics at different parameters in more detail.

\subsection{Influence of noise}

Previous studies of a SCL subject to FOF demonstrated that noise influences the dynamics substantially [21, 23]. They showed that multiple attractors exist for the deterministic dynamics, and in the presence of noise some of the attractors no longer survive 21. The argument followed that noise helps determine whether a particular attractor 


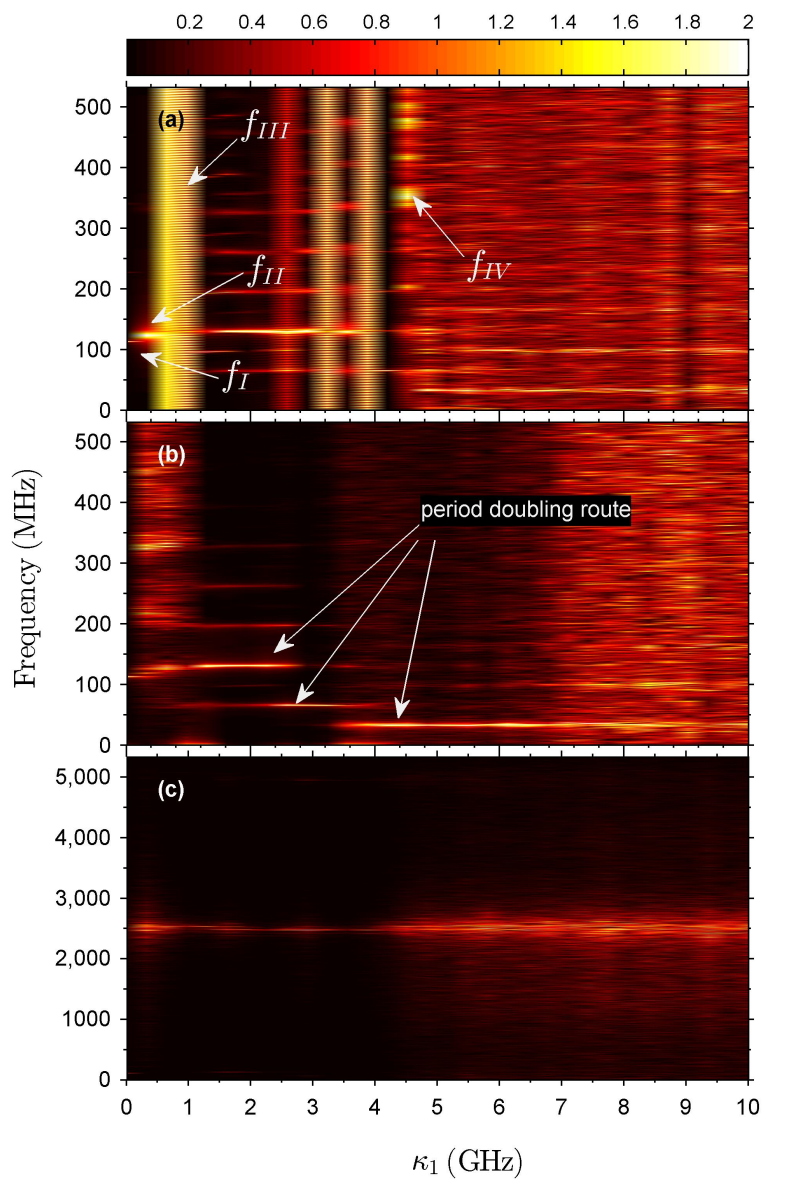

Figure 2: Deterministic (a) and stochastic [(b),(c)] density plots of the period doubling route to chaos when the feedback strength $\kappa_{1}$ from cavity 1 is increased. The bandwidth and detuning are fixed at $\Lambda_{1}=1 \mathrm{GHz}$ and $\Delta=-0.5 \mathrm{GHz}$, otherwise all other parameters are recorded in Table 1 $\mathrm{f}_{\mathrm{I}-\mathrm{IV}}$ are the frequencies discussed in this text which differ significantly in the stochastic period doubling route. Note that (c) contains the stochastic spectra extended out to $5 \mathrm{GHz}$. stable, analogous to that of a perturbation acting on a system at a maximum of a potential distribution. Other studies have shown that noise induces jumps between stable attractors if the ratio of the noise strength to potential barrier is large enough [23]. A good understanding of this behavior is gained via an examination of the time-series in conjunction with the phase-portraits and rf spectra. The previous studies investigated particular instances of a parameter space, while we focus on the period-doubling route to chaos as the feedback strength is increased. Using these tools with the addition of first return maps, the period doubling route is traced along increasing feedback strength $\kappa_{1}$. The return map is generated by determining the maximum values $\left(\omega_{\max }\right)$ for each oscillation in the time series $\omega(t)$. Thus a series of maximum values is generated, and $\omega_{(\max ) n+1}$ is compared to the previous value at $\omega_{(\max ) n}$
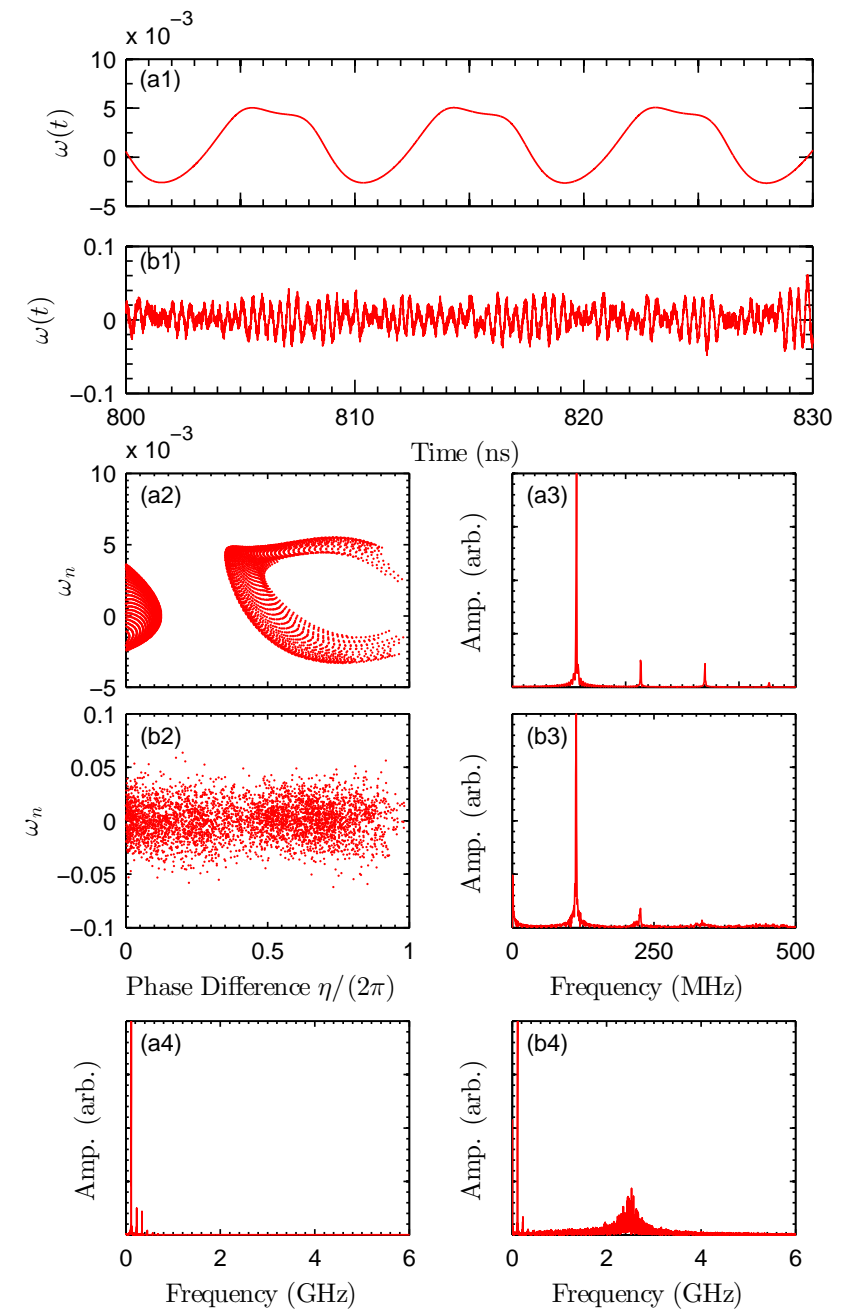

Figure 3: Deterministic (a) and stochastic (b) plots of the frequency $\omega(t)$ time-series (1), the phase-plane of $\omega_{n}$ and $\eta(2)$, and the rf spectrum (3) showing a fundamental frequency of $f_{\text {fun }} \approx 123 \mathrm{GHz}$. The plots are calculated when the feedback is injected solely from cavity $2\left(\kappa_{1}=0 \mathrm{GHz}\right)$. The rf spectra (a4) and (b4) are extended out to $6 \mathrm{GHz}$ in order to depict the undamping of the ROs seen in the time-series (b1). 
Fig. 3 depicts four deterministic plots and four stochastic plots. When the feedback strength is $\kappa_{1}=0 \mathrm{GHz}$, the $\mathrm{e}_{235}$ three corresponding plots are the instantaneous frequency $\omega(t)$ time series (a1 and b1), the phase plane of the frequency $\omega_{n}$ and phase difference $\eta=\phi(t)-\phi(t-\tau)$ (a2 and b2), and the rf spectrum of $\omega(t)$ (a3 and b3). We note, when comparing the deterministic (a3) and stochas-240 tic (b3) rf spectra, that the perturbation of noise does not affect the dominant frequencies in time-delay regime, even though the time-series are clearly different. This difference is noted in the large rf spectra ( $\mathrm{a} 4$ and b4), where in the presence of noise (b4) the ROs are undamped. How-245 ever, as we increase the feedback strength $\kappa_{1}$, noise plays a significant role in determining the dominant frequencies.
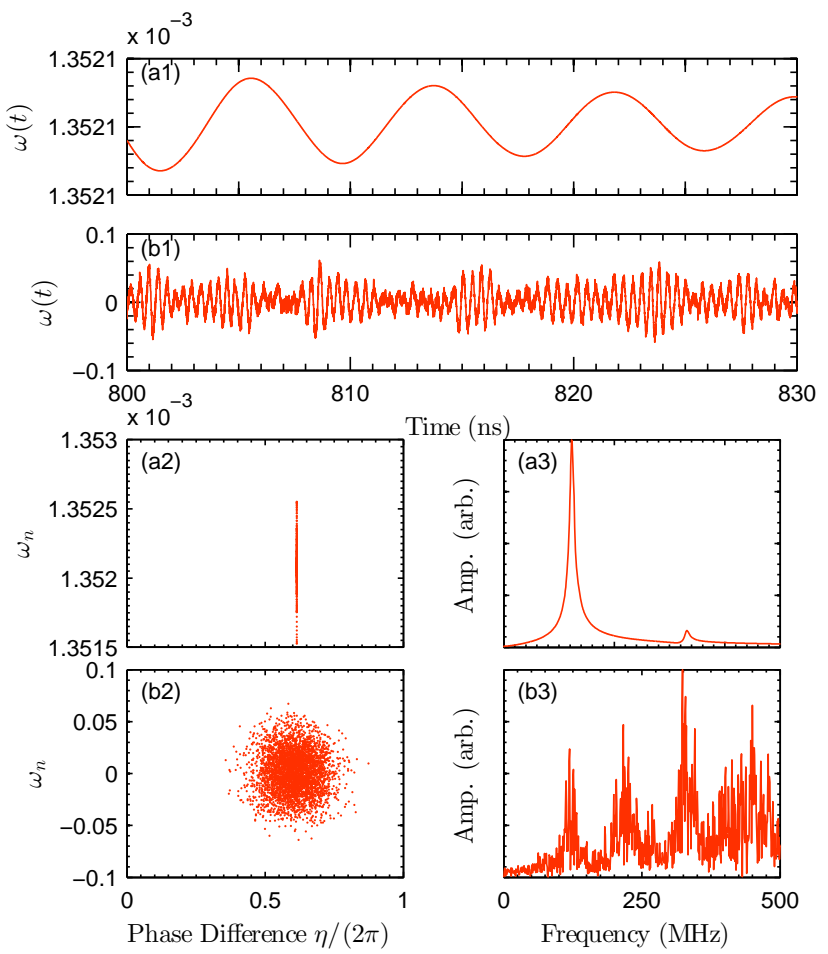

Figure 4: Deterministic (a) and stochastic (b) plots of the frequency $\omega(t)$ time-series (1), the phase-plane of $\omega_{n}$ and $\eta(2)$, and the RIN spectrum (3) when the feedback strength is $\kappa_{1}=0.32 \mathrm{GHz}$. The deterministic time-series captures a transient behavior toward a steadystate behavior.

Once the feedback from the first cavity is allowed $\left(\kappa_{1}=\right.$ $0.32 \mathrm{GHz}$ ), as shown in Fig. 4, the deterministic and stochastic rf spectra [(a3) and (b3)] show clear differences. The differences can be understood by examining the deterministic and stochastic time series [(a1) and (b1)] and $_{265}$ phase portraits [(a2) and (b2)]. From the time series (a1), it is clear that the frequency $\omega(t)$ is always positive and $\omega(t)$ evolves toward a steady-state. The movement toward a steady state (fixed point) is depicted in the deterministic phase portrait (a2). The dominant frequency in the deter-270 ministic spectrum (a3) is $\sim 125 \mathrm{MHz}$. In the presence of noise (b3), the peak at $\sim 125 \mathrm{MHz}$ is reduced and the peak at $\sim 337 \mathrm{MHz}$ is enhanced. In addition, the frequency, shown in the stochastic time series (b1), oscillates around $\omega=0$. We note that this shift in the frequency toward $\omega=0$ is a general feature when noise is included in the simulations. For this feedback strength $(\kappa=0.32 \mathrm{GHz})$, the shift occurs because the fixed point no longer survives in the presence of noise. The absence of the fixed point is clearly depicted when one compares the deterministic and stochastic return maps, shown in Fig.5. Examining the return map (Fig.5), the deterministic maxima (blue circles) show little variance and are centered at the fixed point, while the stochastic maxima (red triangle) spread and are no longer centered at the same location. Instead, noise drives the system toward higher frequencies coinciding with higher harmonics shown in the rf spectrum of Fig. 4(b3).

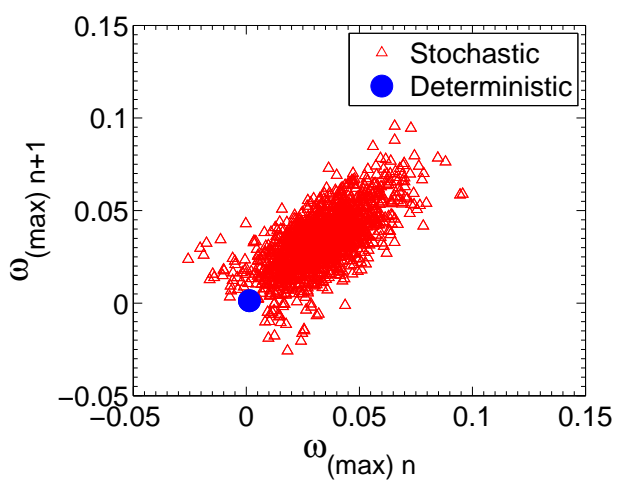

Figure 5: First return maps for feedback strength of $\kappa_{1}=0.32 \mathrm{GHz}$ showing both the stochastic (red triangle) and deterministic (blue circle) locations of $\omega_{\max }$.

At a feedback strength of $\kappa_{1}=0.97 \mathrm{GHz}$, the timeseries in Fig. 6 shows periodic oscillations with a period of $1 / f_{R O}=400 \mathrm{ps}$, where $f_{R O}$ is the frequency of the ROs. This behavior corresponds to the undamping of the ROs, which then become the dominant feature. This undamping of the ROs is a well studied phenomenon of a SCL subject to optical feedback, where the ROs are an exchange of energy between the photons and the inversion carriers when the SCL is externally perturbed [24]. The frequency of these oscillations occur near $f_{R O} \approx 2.5 \mathrm{GHz}$ and varies depending on the SCL design and parameters. The phase portrait [Fig. 6(a2)] shows a periodic attractor, which does not survive in the presence of noise [Fig. 6(b2)]. The extinction of this attractor allows the timedelay oscillations to influence the global behavior seen in the stochastic rf spectrum [Fig. 6(b3)].

Signatures of the ROs in the dynamics occur at three instances along the bifurcation map of Fig. 2(a) at $\kappa_{1}=$ $0.97 \mathrm{GHz}$ (fIII on the map), $\kappa_{1}=3.1 \mathrm{GHz}$, and $\kappa_{1}=3.5$ $\mathrm{GHz}$. Each instance displays the same behavior characterized by frequency oscillations with a period of $1 / f_{R O}=400$ ps and the existence of a limit cycle in the phase-portrait. However, in each case, this attractor no longer survives in the presence of noise. Noise drives the system toward periodic oscillations which are dictated by the delay time 

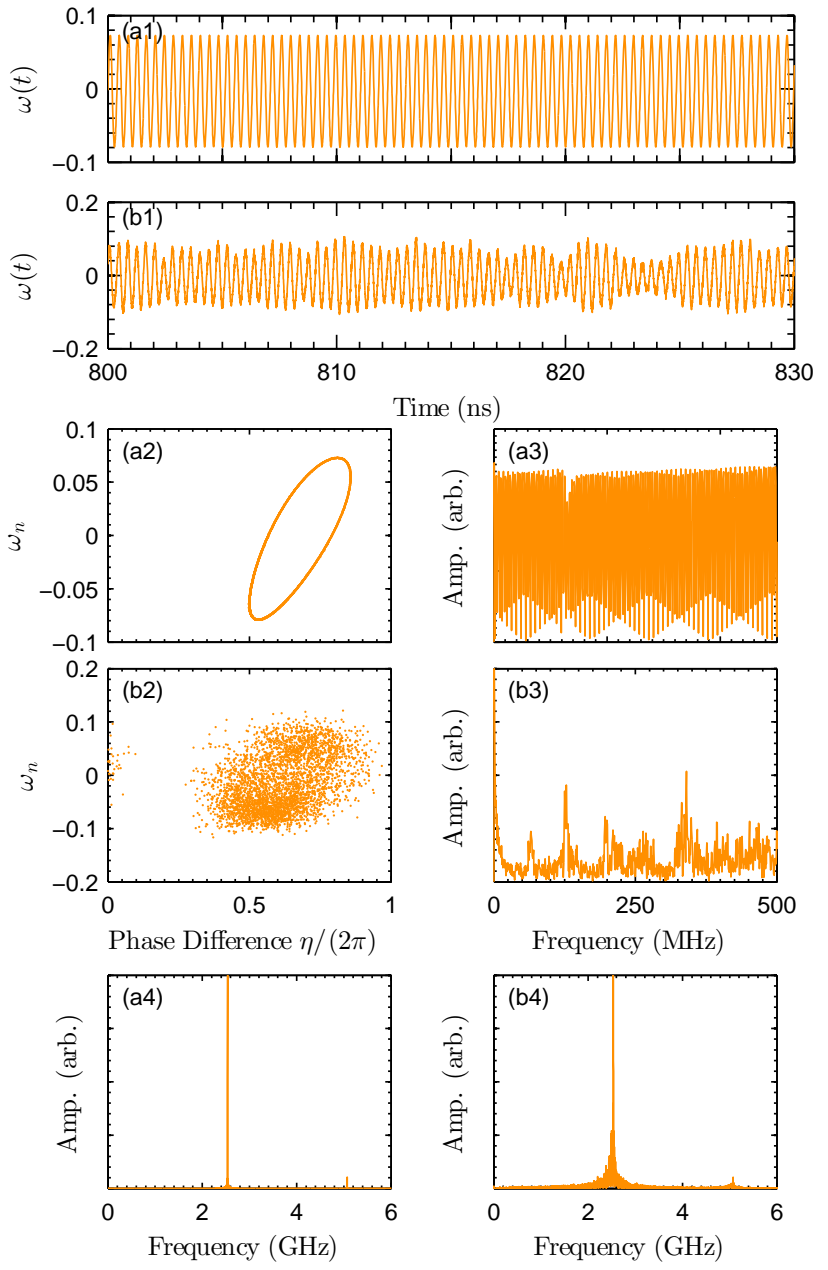

Figure 6: Deterministic (a) and stochastic (b) plots of the frequency ${ }_{295}$ $\omega(t)$ time-series (1), the phase-plane of $\omega_{n}$ and $\eta(2)$, and the $\mathrm{rf}^{295}$ spectrum (3) when the feedback strength is $\kappa_{1}=0.97 \mathrm{GHz}$. The deterministic time-series and phase-portrait show periodic behavior with a frequency of $f_{R O} \approx 2.5 \mathrm{GHz}$, which is shown in the extended spectra (a4) and (b4).

and filter bandwidth.

Turning our attention to the period doubling maps [Fig. 2], we note that the transition into chaotic deterministic dynamics begins at a feedback strength of $\kappa_{1} \approx 4.5$ $\mathrm{GHz}$ ( $\mathrm{f}_{\mathrm{IV}}$ on the map), and in presence of noise the onset of ${ }^{305}$ a chaotic transition is clearly delayed $\left(\kappa_{1} \approx 7.0 \mathrm{GHz}\right)$. Fig.

7 displays this critical transition at a feedback $\kappa_{1}=4.52$

$\mathrm{GHz}$ for the deterministic onset of chaos. The frequency $\omega(t)$ not only oscillates with a period of $1 / f_{R O}$ but exhibits an envelope of slower oscillations which are shown ${ }^{310}$ in the time series (a1) and spectrum (a2) of Fig.77. The return map in Fig. 8(a) suggests stable periodic oscillations demonstrated by the small variance in the deterministic maximum values (blue circle) of the frequency $\omega_{\max }$. Comparing this to the stochastic dynamics, the corresponding ${ }^{315}$ behaviors are very different. Shown in Fig. 7(b2), the attractor no longer survives and the spectrum [Fig. 7(b3)] greatly differs. The spectrum contains a period doubled
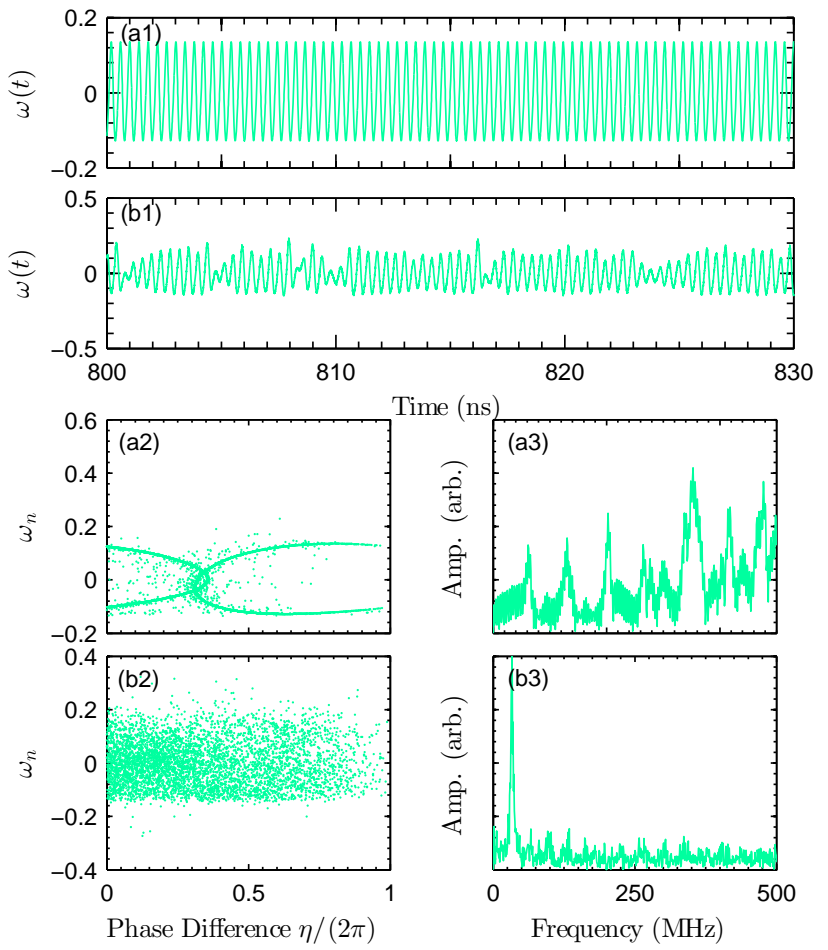

Figure 7: Deterministic (a) and stochastic (b) plots of the frequency $\omega(t)$ time-series (a1 and b1), the phase-plane of $\omega_{n}$ and $\eta$ (a2 and $\mathrm{b} 2$ ), and the rf spectrum (a3 and b3) when the feedback strength is $\kappa_{1}=4.52 \mathrm{GHz}$.

frequency located at $f_{d o m} / 2=62 \mathrm{MHz}$. Here we define $f_{d o m}$ as the dominant frequency which corresponds to the weighted average between the two fundamental frequencies of each cavity. Once again, the dominant frequency in the presence of noise is dictated by the FOF time scales $(\tau$ and $\Lambda)$.

The return maps in Fig. 8 for feedback strengths $\kappa_{1}=4.84$ GHz [Fig. 8(b)] and $\kappa_{1}=5.16 \mathrm{GHz}$ [Fig. 8(c)] provide an excellent representation of the difference between the deterministic and stochastic dynamical states. Comparing all three return maps [Fig. 8 $8, b$, and c], the stochastic maxima (red triangles) reveal a departure from the linear shape at smaller feedback strengths (see Fig.5). The deterministic maxima (blue circles) $\omega_{\max }$, however, require a larger feedback strength $\left(\kappa_{1}=5.16 \mathrm{GHz}\right)$ in order to replicate stochastic maps. It is not until $\kappa_{1}=5.16 \mathrm{GHz}$ [Fig. 8(c)] that the two maps become similar.

However, the similarity in the return maps [Fig. 8(c)] does not guarantee that the dynamical states will be identical. The difference between the stochastic and deterministic dynamics is manifested in the period doubling routes shown in Fig. 2, where two key differences standout. First, the lack of stable RO frequencies in Fig. 2(b) (stochastic), which are present in Fig.2(a) (deterministic). An example of the RO frequency in Fig.2(a) is displayed by the marker $\mathrm{f}_{\mathrm{III}}$. Second, the onset of chaos is delayed in Fig. 2(b) until a feedback strength of $\kappa_{1} \approx 7 \mathrm{GHz}$ is reached. To explain these differences, it appears that multiple mecha- 

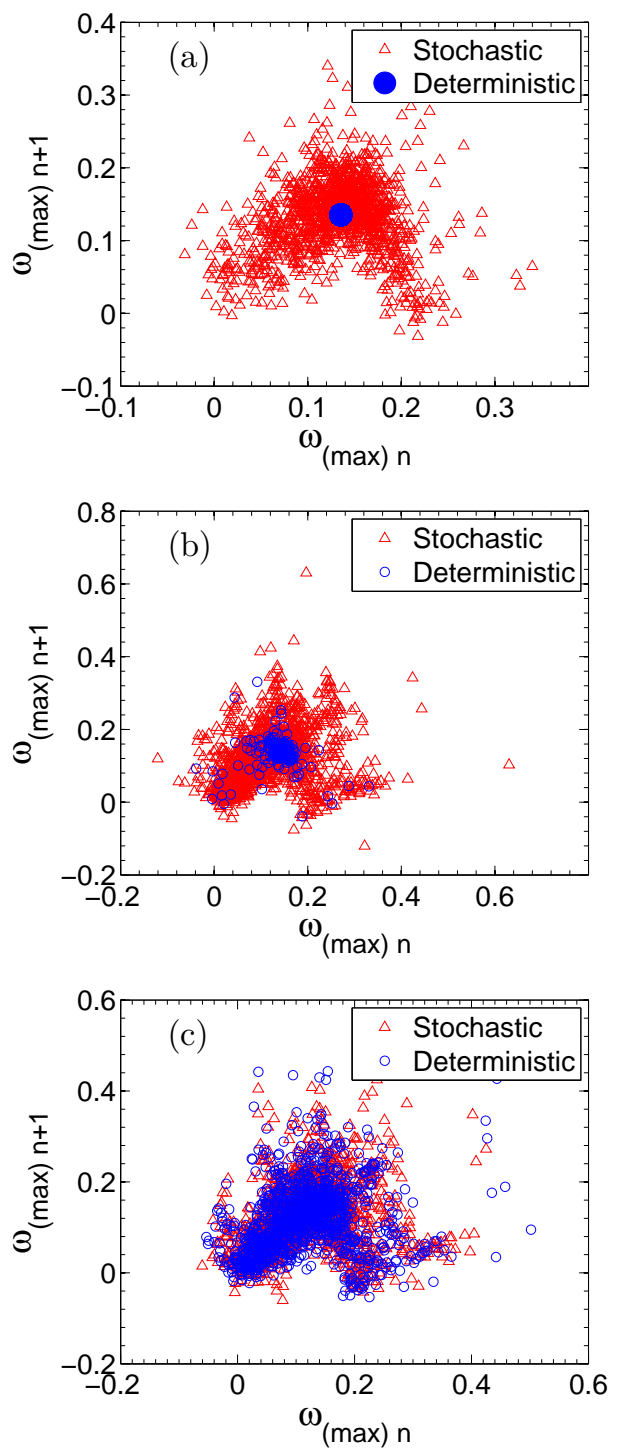

Figure 8: First return maps for feedback strength of (a) $\kappa_{1}=4.52$ $\mathrm{GHz}$, (b) $\kappa_{1}=4.84 \mathrm{GHz}$, and (c) $\kappa_{1}=5.16 \mathrm{GHz}$ showing both the $\mathrm{B}_{370}$ stochastic (red triangle) and deterministic (blue circle) values.

nisms play a role. Not only does noise limit the number of stable attractors, but noise drives the periodic oscillations toward the dynamics influenced by the time-delay ${ }^{375}$ and filter bandwidth.

\section{Effects of the filter bandwidth on the dynamics}

We next turn our attention to the effects of the two ${ }^{380}$ filter bandwidths on the dynamics. In a single FOF system, it is possible to identify three regimes depending on the magnitude of the bandwidth $\Lambda$ relative to the RO frequency $f_{R O}$ and relative to the external cavity mode (ECM) spacing. The effects of the filter width on the dynamics for each case have been outlined by Fischer [25], while other theoretical work has characterized the mode structures for the different bandwidth regimes [26]. Our

previous bifurcation analysis was done for bandwidths of intermediate range. The intermediate range is where $\Lambda$ is large enough to include a number of ECMs yet smaller than the frequency of the ROs. A narrow filter $(\Lambda \rightarrow 0)$ was shown to resemble optical injection [27, while a wide cal feedback (COF), hence the latter case reduces to the standard Lang-Kobayashi rate equations [26]. In general, the most interesting dynamics occur in the intermediate regime, because in this regime the parameters of the filter stability and control of the dynamics 25 .

The use of two filters is advantageous not only because of the additional parameters introduced $\left(\tau_{2}, \Lambda_{2}, \Delta_{2}\right.$, etc.) but also because of the interplay between the two fields results in novel dynamics and the possibility of robust control over these dynamics 18. As stated earlier, we fix one filter $\Lambda_{1}$ in the intermediate regime while the other filter $\Lambda_{2}$ is varied. In particular, we focus on two cases. The first is a narrow filter $\Lambda_{2}<\delta_{E C M}$, where ing. The second is a wide filter, $\Lambda_{2}>f_{R O}$. We achieve this by varying the bandwidth of one filter over a large range from $\Lambda_{2}=0 \rightarrow 20 \mathrm{GHz}$, while the second filter is fixed at $\Lambda_{2}=1 \mathrm{GHz}$. We focus on these two cases in order to isolate the effects of both noise and the bandwidth. A study could be done of the narrow-narrow, wide-wide, or narrow-wide cases, but these would no longer contain any signatures of the spectrally filtered feedback. Rather, they would resemble injection and COF.

\subsection{Feedback from an intermediate and narrow filter}

In practice, one is not able to reduce the bandwidth indefinitely, therefore we restrict ourselves to the narrow filter where $0<\Lambda<\delta_{E C M}$. Experimentally, the bandwidth of the filter can be modified by changing the mirror reflectivity of the filter or the mirror spacing. We study this system by keeping $\Lambda_{2}=1 \mathrm{GHz}$ while $\Lambda_{1}$ and $\Delta_{1}$ are varied from $10 \mathrm{MHz}$ to $50 \mathrm{MHz}$ and $-10 \mathrm{MHz}$ to $-50 \mathrm{MHz}$, respectively, where $50 \mathrm{MHz}$ is slightly larger than the SCL linewidth. Along each iteration of the bandwidth, the detuning is varied such that the laser frequency lies at the same position of the filter profile. Similar to the period doubling map, the spectra are stitched together for different $\Lambda_{1}$ and Fig. 9 shows the resultant density plots of the intensities (1) $I_{L}(t),(2) I_{F_{1}}(t)$, and $(3) I_{F_{2}}(t)$, where $I_{L}(t)=|E(t)|^{2}, I_{F_{1}}(t)=\left|F_{1}(t)\right|^{2}$, and $I_{F_{2}}(t)=\left|F_{2}(t)\right|^{2}$.

Initially, for the deterministic case, when $\Lambda_{1}$ is small ( $0 \mathrm{MHz}<\Lambda_{1}<20 \mathrm{MHz}$ ), the frequency of oscillations is $f_{\text {dom }} \approx 110 \mathrm{MHz}$ shown in Figure 9(a). This frequency of $110 \mathrm{MHz}$ corresponds to the fundamental frequency of cavity 2 . Using the parameters in Table 1 , we find that $f_{2} \approx 1 /\left(\tau_{2}+1 / \Lambda_{2}\right) \approx 112 \mathrm{MHz}$. When $\Lambda_{1}$ is increased, a frequency of $50 \mathrm{MHz}$ emerges, which is a consequence of the spectrally filtered feedback from cavity 1 . The frequency of $50 \mathrm{MHz}$ is an average between $f_{1}$ and $f_{2}$, where 


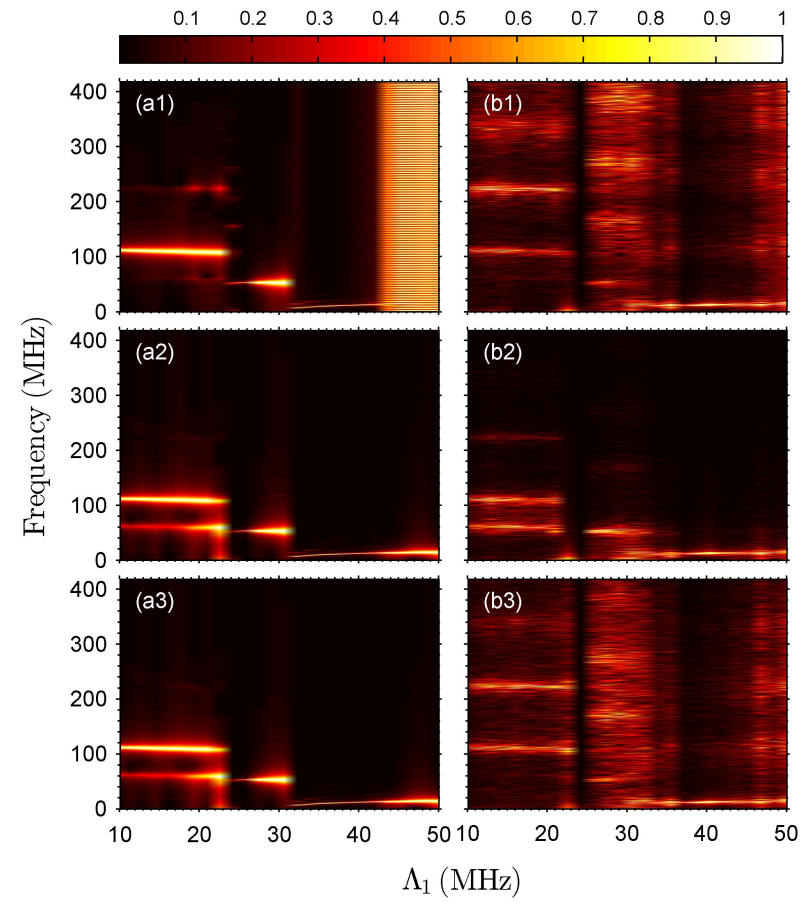

Figure 9: Deterministic (a) and stochastic (b) density plots of the amplitude of the spectrum as a function of changing bandwidth, $\Lambda_{1}$. The density plots correspond to the spectra for $(1) I_{L}(t),(2) I_{F_{1}}(t)$, (3) and $I_{F_{2}}(t) . \Lambda_{2}$ is fixed at $1 \mathrm{GHz}$ and the two feedback rates are kept constant at $\kappa_{1}=3 \mathrm{GHZ}$ and $\kappa_{2}=0.8 \mathrm{GHz}$.

$f_{1} \approx 1 /\left(\tau_{1}+1 / \Lambda_{1}\right) \approx 15 \mathrm{MHz}$. The average, weighted toward $f_{1}$, is explained by the larger feedback rate from cavity $1\left(\kappa_{1}>\kappa_{2}\right)$. The dependence of the weighted average on the ratio of the feedback rates was reported in Ref. [18. Continuing to increase $\Lambda_{1}$ results in a frequency of approximately $15 \mathrm{MHz}$, which is dominated by $f_{1}$. Upon further increase of $\Lambda_{1}$ the ROs become dominant which is evident in the time series plot in Fig. 11(a1). Note that these ROs are not present in Figs. 9 (a2) and 9(a3) because, $\Lambda_{1}$ and $\Lambda_{2}$ act as low pass filters. The same density plots are then produced in the presence of noise shown in Fig. 9b. For all three plots in Fig. 9b, there is an absence of the broad $50 \mathrm{MHz}$ frequency at a bandwidth of $\Lambda_{1}=32$ MHz.

The deterministic time series for $\Lambda_{1}=32 \mathrm{MHz}$ in Fig. 10(a1) shows the evolution of $I_{L}(t)$ toward a steadystate value and the phase portrait (a2) shows this fixed point attractor. Comparing this to the stochastic time series for $\Lambda_{1}=32 \mathrm{MHz}$ in Fig. 10(b1), it becomes evident that noise drives the system out of the fixed point, which was explained in our analysis above describing the period-doubling route to chaos. Comparing Fig.11(a1) and Fig.11(b1), it is clear that the relaxation oscillations are no longer the dominant frequency for the deterministic case at a larger bandwidth of $\Lambda_{1}=50 \mathrm{MHz}$, rather the dominant frequency contains signatures of the FOF time scale in the rf spectrum shown in Fig 11(b3).

Although $\Lambda_{1}<\delta_{E C M}$, we point out that the feedback

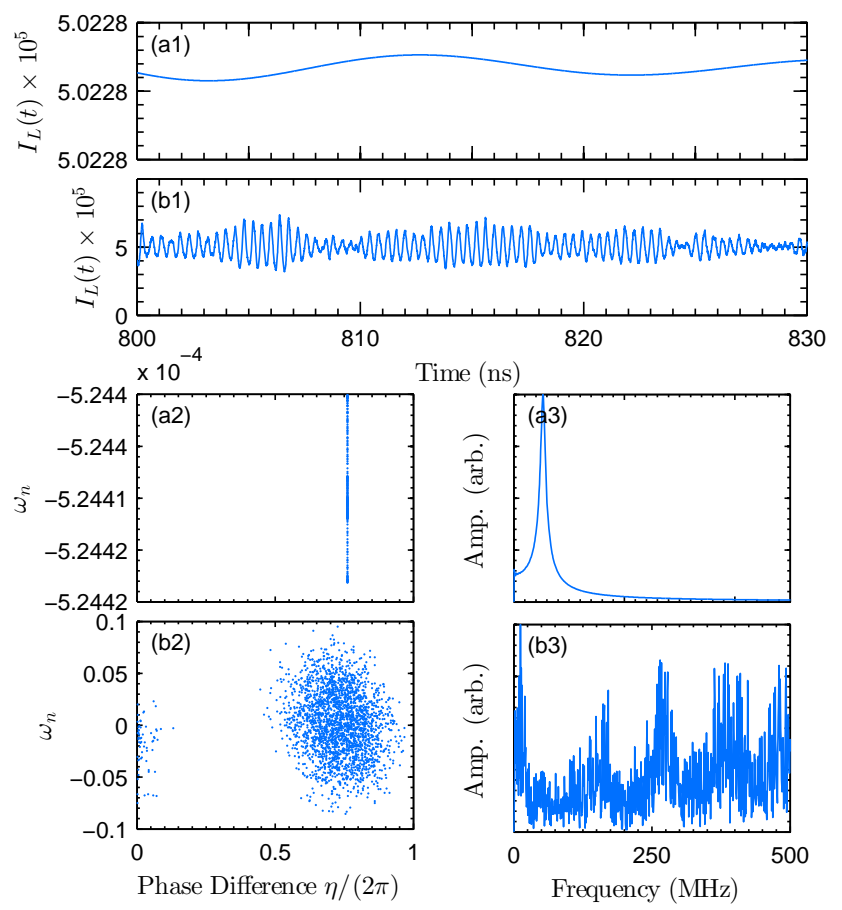

Figure 10: (1) Intensity time series $I_{L}(t),(2)$ phase portraits $(\eta, P)$, and (3) rf spectrum in the absence of noise (a) and in the presence of noise (b) for a bandwidth of $\Lambda_{1} \approx 32 \mathrm{MHz}$ and detuning $\Delta_{1}=-32$ $\mathrm{MHz}$.
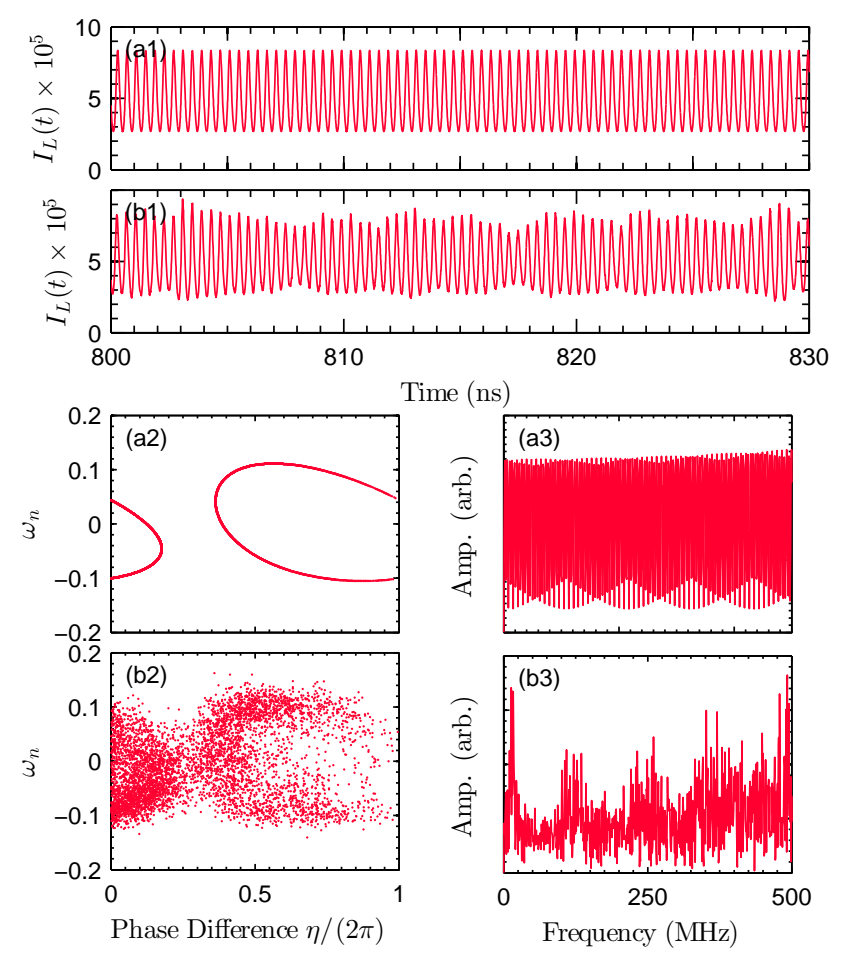

Figure 11: (1) Intensity time series $I_{L}(t),(2)$ phase portraits $\left(\omega_{n}, \eta\right)$, and (3) rf spectrum in the absence of noise (a) and in the presence of noise (b) for a bandwidth of $\Lambda_{1}=50 \mathrm{MHz}$ and detuning $\Delta_{1}=-50$ $\mathrm{MHz}$. 
from cavity 1 only resembles optical injection when the bandwidth is $\Lambda_{1}<25 \mathrm{MHz}$. This is observed by notwhen $\kappa_{2}=0 \mathrm{GHz}$ (not shown), evolve to a steady-state fixed point for any bandwidth of $\Lambda_{1}$ up to $25 \mathrm{MHz}$. Beyond a bandwidth of $\Lambda_{1}=25 \mathrm{MHz}$ the dynamics become periodic and the period of these dynamics depend cal injection. In summary, both the narrow-intermediate and intermediate-intermediate cases show that particular attractors no longer survive and, in turn, drive the system toward periodic oscillations determined by the a FOF time particular feedback rates $\kappa_{1}$ and $\kappa_{2}$. A complete picture becomes highly complex due to the amount of parameters $\Lambda_{1,2}, \tau_{1,2}, \Delta_{1,2}, \kappa_{1,2}$ which influence the dynamical behavior of this system.

\subsection{Feedback from an intermediate and wide filter}

We next investigate the effects of a competition between an intermediate filter and wide filter. The dynamics of both cases have been intensely studied for the single FOF case which showed that the stability and dynamics

440 vary depending on the filter width [25. Knowing that the wide filter resembles COF, we expect to see only two time ${ }^{475}$ signatures $\left(\tau\right.$ and $1 / f_{R O}$ ), instead of the influence of the bandwidth $\Lambda$ found in FOF dynamics.

To study this system, we fix both bandwidths at $\Lambda_{1}=$ $20 \mathrm{GHz}$ and $\Lambda_{2}=1 \mathrm{GHz}$ and detunings $\Delta_{1}=-5 \mathrm{GHz}$ and $\Delta_{2}=-1.5 \mathrm{GHz} . \kappa_{1}$ is varied while $\kappa_{2}=0.8 \mathrm{GHz}$ is ${ }^{480}$ fixed. $\kappa_{1}$ is varied, rather than $\Lambda_{1}$, because the period of elicited oscillations for this two FOF system remain unaltered when $\Lambda_{1}$ is increased. Fig.12 contains the spectra of the intensity of the laser $I_{L}(t)$ and the intensity of the light through filter $2, I_{F_{2}}(t)$, when the feedback rate $\kappa_{1}{ }^{485}$ is varied. These two intensities were chosen because the light through filter $1, I_{F_{1}}$, directly mimics $I_{L}(t)$ due to the wide filter and the presence of the ROs, which are not as dominant in the dynamics of feedback from filter 2 .

Initially, the feedback is solely from cavity $2\left(\kappa_{1}=0^{490}\right.$ $\mathrm{GHz}$ ). Therefore, the dynamics which arise are a consequence of the spectrally filtered (intermediate) feedback from cavity 2, which are shown in Fig. 13 for feedback rates of $\kappa_{2}=0.8 \mathrm{GHz}$ and $\kappa_{1}=0 \mathrm{GHz}$. It is clear that the rf spectra of the deterministic (a3) and stochastic (b3) ${ }^{495}$ dynamics are very similar at this feedback strengths. The dominant frequency $f_{2} \approx 112 \mathrm{MHz}$ corresponds to the fundamental frequency of cavity 2 . The higher harmonics are also present with decreasing amplitudes. The feedback rate $\kappa_{1}$ is increased which enhances the second and ${ }^{500}$ third harmonic shown in both Fig. 12(a1) and (a2). As the feedback rate increases, $\kappa_{1} \approx 0.32 \mathrm{GHz}$, a frequency at $f \approx 66 \mathrm{MHz}$ emerges. We note that this corresponds to the fundamental frequency from cavity 1 which is $f_{1} \approx 1 / \tau_{1}$, which has no signatures of the bandwidth $\left(\Lambda_{1}=20 \mathrm{GHz}\right) .{ }^{505}$ A continued increase in $\kappa_{1}$ results in the enhancement of

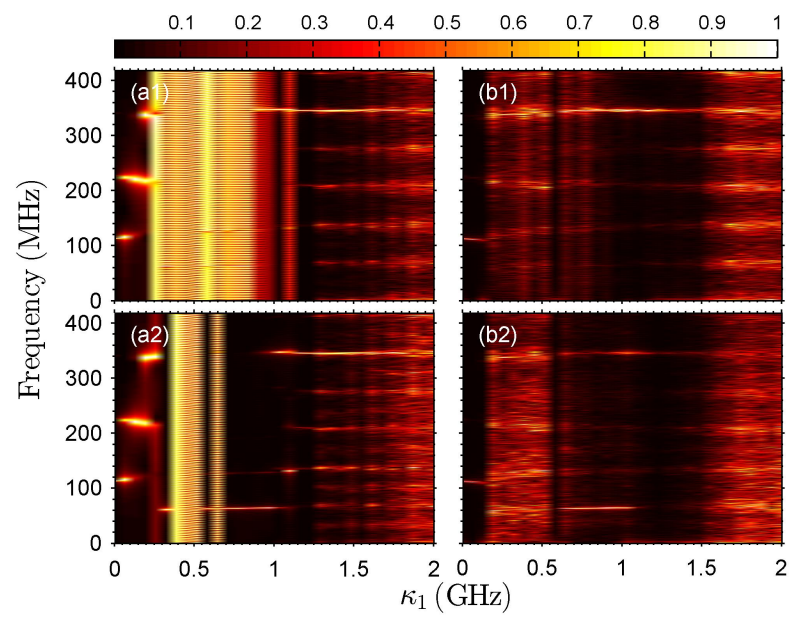

Figure 12: Deterministic (a) and stochastic (b) density plots of the amplitude of the spectrum as a function of the increasing feedback rate, $\kappa_{1}$. The spectra are calculated with the with the parameters mentioned in Table 1 except the following: $\Lambda_{1}=20 \mathrm{GHz}$ and $\Delta_{1}=$ $-5 \mathrm{GHz}$. (1) Spectra of the intensity of light from laser, $I_{L}(t)$ and (2) spectra from the light through filter $2, I_{F_{2}}(t)$.

the successive higher harmonics, the second and third harmonic emerge, $2 f_{1}$ and $3 f_{1}$ respectively. The ROs appear in the laser intensity, which is shown Fig.12(a1), while they are attenuated through filter 2 shown in Fig. 12(a2).

The dynamics become more complicated and eventually evolve into the chaotic regime as the feedback rate $\kappa_{1}$ is increased. This is seen in all three subplots in Fig. $13(\mathrm{c} 1)$ and Fig $13(\mathrm{~d} 1)$ at feedback rate of $\kappa_{1}=1.7 \mathrm{GHz}$. The complicated time series, the jumping between attractors in the phase portrait and the spread in the rf spectrum all indicate the emergence of a chaotic regime. This behavior is well known for COF systems as the feedback rate is increased eventually inducing coherence collapse.

Comparing the deterministic [Fig. 12(a)] and stochastic [Fig. 12(b)] spectra (density plots) for an increasing feedback strength $\left(\kappa_{1}\right)$, it is evident that noise plays a significant role in determining which frequencies emerge. This is understood by noting that the filter widths are larger, thus effectively reducing the spectral filtering of the feedback fields. Initially, the frequency spectra are very similar when $\kappa_{1}=0 \mathrm{GHz}$ shown in Fig 13(a3) and Fig. 13(b3). As $\kappa_{1}$ is increased, two regions of dissimilarity emerge. The first is a lack of the second and third harmonic found in Fig. 12(b). Upon further investigation we found that these harmonics emerge as the system evolves toward a steady state intensity. When the feedback rate is $0.1 \mathrm{GHz}$ $<\kappa_{1}<0.3 \mathrm{GHz}$, noise drives the system out of these fixed point solutions, which is in good agreement with the previous analysis.

However, it is interesting to note that noise does not appear to delay the onset of chaos in larger feedback regimes $\left(\kappa_{1}>1.5 \mathrm{GHz}\right)$ shown in Fig. 12(b). A stark difference between the former bandwidth regimes and the wideintermediate regime is that there is no averaging between 

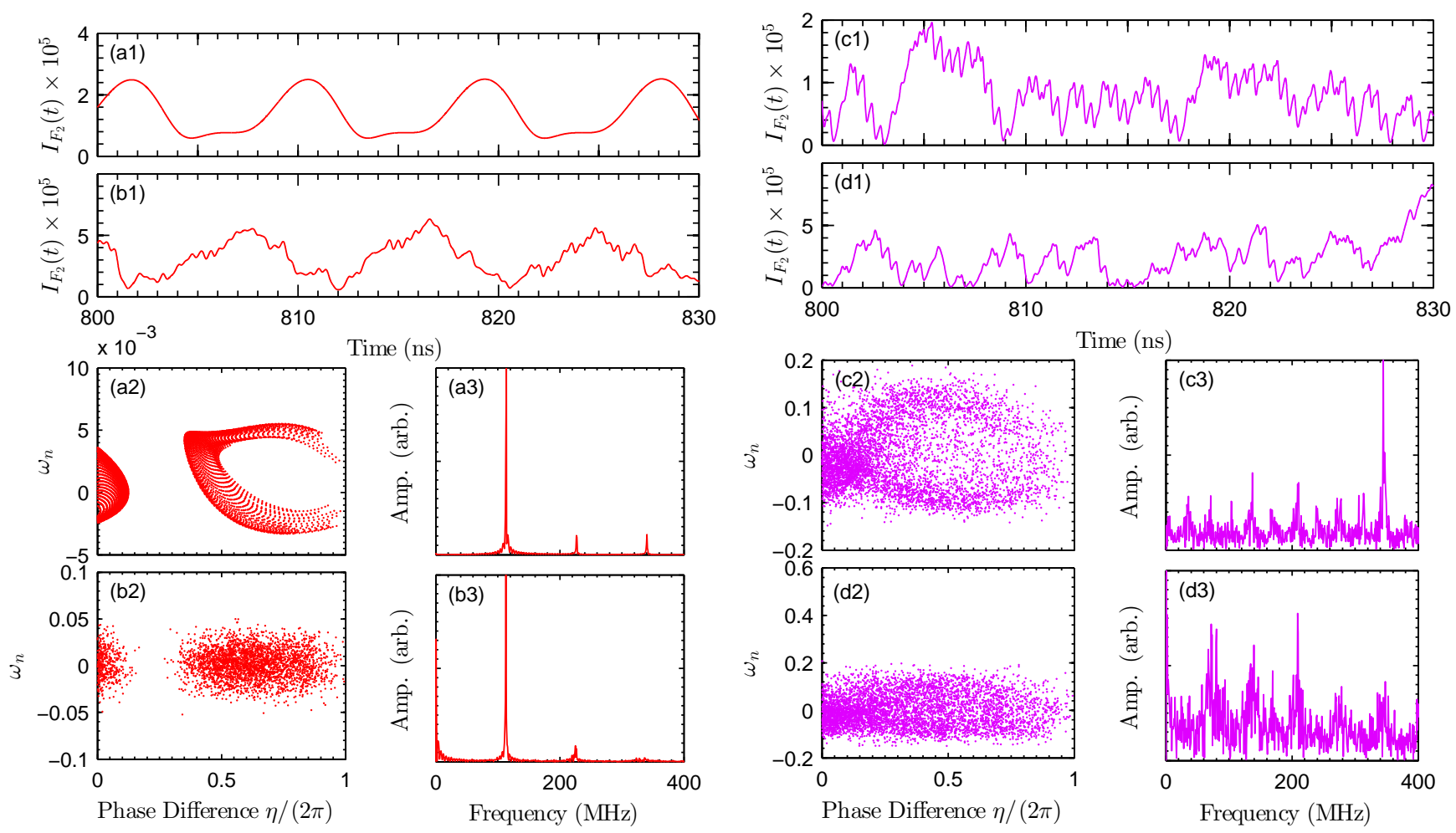

Figure 13: Three subplots are produced using the intensity of light through filter $2, I_{F_{2}}(t)$. The times series $(1)$, phase portrait $\omega_{n}, \eta(t)(2)$, and rf spectrum (3) are calculated for the feedback rates $[(\mathrm{a})$ and $(\mathrm{b})] \kappa_{1}=0 \mathrm{GHz}$ and $[(\mathrm{c})$ and $(\mathrm{d})] \kappa_{1}=1.7 \mathrm{GHz}$.

the fundamental frequencies of the cavities, i.e. the dynamics are dictated by cavity 2 (intermediate filter) or cavity 1 (wide filter). In the presence of noise, two chaotic535 (n) emerge, when $0.3 \mathrm{GHz}<\kappa_{1}<0.55 \mathrm{GHz}$ and $\kappa_{1}>1.7 \mathrm{GHz}$ [Fig. 12(b)]. For small feedback strengths $\left(0.3 \mathrm{GHz}<\kappa_{1}<0.55 \mathrm{GHz}\right)$ when the RO dynamics emerge [Fig. 12(a)], instead of driving the system toward periodic oscillations, the dynamics become chaotic [Fig.540 515 12(b)]. In summary, the presence of a large bandwidth does not filter the frequency content and thus permits chaotic dynamics when the deterministic periodic (ROs) attractors no longer survive in the presence of noise even for a smaller feedback rate $\left(0.3 \mathrm{GHz}<\kappa_{1}<0.55 \mathrm{GHz}\right)$. ${ }_{545}$

\section{Discussion and conclusion}

In this paper, we have reported on a theoretical and computational investigation of the effects of quantum noise ${ }^{550}$ on the complex dynamics that arise in the instantaneous optical frequency of a SCL that is subject to two filtered optical feedbacks. A majority of the results deal with the situation where the bandwidths of both filters through which the feedbacks are filtered are in the intermediate ${ }^{555}$ regime and wherein the feedback strength from one cavity is kept fixed while the feedback strength from the second cavity is varied. For these parameters, the most important observation is that the feedback strength needed from the second cavity to produce coherence collapse is significantly ${ }^{560}$ increased in the presence of noise. Our calculations indicate that this is a general feature of two FOFs in the presence of noise, even though we have displayed the results for one set of parameters. The physical origin of this increased feedback needed to produce coherence collapse lies in the fact that some of the attractors in the deterministic model do not survive in the presence of noise. An important consequence of this is that the effects of the time-delay and filter bandwidth are enhanced in the presence of quantum noise.

Most of the results that we report show the dynamics up to frequencies of a few hundred $\mathrm{MHz}$ since these can be easily measured with standard detection components with rise times of a few nanoseconds. In some representative cases we also show the observed behaviors extended out to a few $\mathrm{GHz}$, and the dominant theme to emerge from these data is that as the feedback strength from the second cavity is increased, the relaxation oscillations become the more dominant feature. In general, the results indicate that the effect of noise is to destroy the periodic attractor related to the relaxation oscillations and to enhance the effects of time-delay and filter bandwidth.

Typically, the effects of quantum noise will be most prominent when both filters have bandwidths in the intermediate regime. For narrow filters, any relaxation oscillations that are undamped will be suppressed by the narrow filters and hence the dynamics will be dominated by the time-delay of the feedbacks. For wide filters, signatures 
of the time-delayed feedback will be suppressed by the re-615 laxation oscillations. It is only the intermediate regime in which noise can push the dynamics either towards making the time-delay dynamics the dominant ones or the relaxation oscillations the dominant ones, depending on feed-620 back strengths.

This paper also reports the laser frequency dynamics when one of the filters has an intermediate bandwidth and the other is either much narrower or much wider. In the625 former case, the effect of quantum noise is to once again drive the system away from the deterministic fixed point. In addition, the relaxation oscillations are suppressed and the time scale associated with the time-delayed feedback ${ }^{630}$ and the filter bandwidth contribute to the dominant frequencies. In the case of one intermediate filter and one wide filter, the spectral filtering of the feedback is reduced and as a consequence one finds that the laser frequency ${ }^{635}$ goes into the coherence collapse regime at about the same feedback levels in the presence of quantum noise as it does in the deterministic case. Therefore, it is clear that the requirement for a higher feedback strength to induce co-640 herence collapse in the presence of quantum noise arises only when the filter bandwidths are in the intermediate regime, even though noise does play a role in determining which deterministic attractors persist.

In summary, our work confirms that it is important to include the role of quantum noise to accurately describe the frequency dynamics of a SCL subject to two FOFs. The complexity and richness of this multi-parameter sys- ${ }^{650}$ tem raises some interesting and important questions, such as the physical mechanisms that cause laser frequency dynamics to be dominated by the time-delay and filter bandwidth in the two intermediate filter case, the reasons for ${ }^{655}$ the return maps to deviate from a linear shape in the presence of noise, the effects of correlated amplitude and inversion noise, and the influence of different noise strengths and of other combinations of filter widths. We hope to ${ }^{660}$ explore these, and other, questions in future studies.

\section{Acknowledgments}

This research was supported in part by Lilly Endowment, Inc., through its support for the Indiana University Pervasive Technology Institute, and in part by the Indiana ${ }_{670}$ METACyt Initiative. The Indiana METACyt Initiative at $\mathrm{IU}$ is also supported in part by Lilly Endowment, Inc.

\section{References}

[1] M. C. Soriano, J. García-Ojalvo, C. R. Mirasso, I. Fischer, Complex photonics: Dynamics and applications of delay-coupled semiconductors lasers Rev. Mod. Phys. 85 (2013) 421-470. doi:10.1103/RevModPhys.85.421

URL http://link . aps . org/doi/10.1103/RevModPhys .85.421

[2] M. Sciamanna, K. Shore, Physics and applications of laser diode chaos, Nature Photonics 9 (3) (2015) 151-162.

[3] M. C. Soriano, S. Ortín, D. Brunner, L. Larger, C. R. Mirasso, I. Fischer, L. Pesquera, Optoelectronic reservoir computing: ${ }^{\beta 85}$ tackling noise-induced performance degradation Opt. Express 21 (1) (2013) 12-20. doi:10.1364/0E.21.000012.

I URL http://www.opticsexpress.org/abstract.cfm?URI= oe-21-1-12

[4] M. Peil, M. Jacquot, Y. K. Chembo, L. Larger, T. Erneux, Routes to chaos and multiple time scale dynamics in broadband bandpass nonlinear delay electro-optic oscillators Phys. Rev. E 79 (2009) 026208. doi:10.1103/PhysRevE.79.026208 URL http://link .aps.org/doi/10.1103/PhysRevE.79.026208

[5] R. Lang, K. Kobayashi, External optical feedback effects on semiconductor injection laser properties, Quantum Electronics, IEEE Journal of 16 (3) (1980) 347-355. doi : 10.1109/JQE. 1980. 1070479

[6] D. Lenstra, B. Verbeek, A. Den Boef, Coherence collapse in single-mode semiconductor lasers due to optical feedback, Quantum Electronics, IEEE Journal of 21 (6) (1985) 674-679. doi:10.1109/JQE.1985.1072725.

[1] T. Heil, A. Uchida, P. Davis, T. Aida, Te-tm dynamics in a semiconductor laser subject to polarization-rotated optical feedback Phys. Rev. A 68 (2003) 033811. doi:10.1103/PhysRevA. 68.033811

URL http://link .aps .org/doi/10.1103/PhysRevA.68.033811

[8] N. Oliver, M. C. Soriano, D. W. Sukow, I. Fischer, Dynamics of a semiconductor laser with polarization-rotated feedback and its utilization for random bit generation Opt. Lett. 36 (23) (2011) 4632-4634. doi:10.1364/0L.36.004632.

URL http://ol.osa.org/abstract. cfm?URI=ol-36-23-4632

[9] G. Friart, G. Verschaffelt, J. Danckaert, T. Erneux, All-optical controlled switching between time-periodic square waves in diode lasers with delayed feedback Opt. Lett. 39 (21) (2014) 6098-6101. doi:10.1364/0L.39.006098.

URL http: //ol .osa.org/abstract.cfm?URI=ol-39-21-6098

[10] G. Friart, A. Gavrielides, T. Erneux, Analytical stability boundaries of an injected two-polarization semiconductor laser, Phys. Rev. E 91 (2015) 042918. doi:10.1103/PhysRevE.91.042918. URL http://link .aps .org/doi/10.1103/PhysRevE.91.042918

[11] M. Yousefi, D. Lenstra, Dynamical behavior of a semiconductor laser with filtered external optical feedback, Quantum

Electronics, IEEE Journal of 35 (6) (1999) 970-976. doi: 10.1109/3.766841

[12] A. P. A. Fischer, M. Yousefi, D. Lenstra, M. W. Carter, G. Vemuri, Filtered optical feedback induced frequency dynamics in semiconductor lasers Phys. Rev. Lett. 92 (2004) 023901. doi:10.1103/PhysRevLett.92.023901

11 URL http://link.aps.org/doi/10.1103/PhysRevLett.92. 023901

[13] H. Erzgräber, B. Krauskopf, D. Lenstra, A. P. A. Fischer, G. Vemuri, Frequency versus relaxation oscillations in a semiconductor laser with coherent filtered optical feedback Phys. Rev. E 73 (2006) 055201. doi:10.1103/PhysRevE.73.055201 URL http://link.aps.org/doi/10.1103/PhysRevE.73.055201

[14] L. Jaurigue, E. Sch oll, K. L udge, Passively mode-locked laser coupled to two external feedback cavities (2015). doi:10.1117/ 12.2076118

URL http://dx.doi.org/10.1117/12.2076118

[15] P. Slowiński, B. Krauskopf, S. Wieczorek, Mode structure of a semiconductor laser with feedback from two external filters, Discrete and Continuous Dynamical Systems - Series B 20 (2) (2015) 519-586. doi:10.3934/dcdsb.2015.20.519

URL

http://aimsciences.org/journals/ displayArticlesnew . jsp?paperID=10722

[16] P. Slowiński, B. Krauskopf, S. Wieczorek, Mode structure of a semiconductor laser with feedback from two external filters (2008). doi:10.1117/12.780576.

URL http://dx.doi.org/10.1117/12.780576

[17] P. Slowiński, B. Krauskopf, S. Wieczorek, Solution structure

1. and dynamics of a semiconductor laser subject to feedback from two external filters (2010). doi:10.1117/12.853448. URL http://dx.doi.org/10.1117/12.853448

[18] V. Pal, J. Suelzer, A. Prasad, G. Vemuri, R. Ghosh, Semiconductor laser dynamics with two filtered optical feedbacks, 
Quantum Electronics, IEEE Journal of 49 (3) (2013) 340-349. doi:10.1109/JQE.2013.2244559.

[19] G. Agrawal, N. Dutta, Long wavelength semiconductor lasers, Van Nostrand Reinhold Co. Inc.,New York, NY, 1986.

$690[20]$ G. Gray, R. Roy, Noise in nearly-single-mode semiconduc-

(1) tor lasers Phys. Rev. A 40 (1989) 2452-2462. doi:10.1103/ PhysRevA.40.2452

URL http://link .aps.org/doi/10.1103/PhysRevA .40.2452

[21] M. Yousefi, D. Lenstra, G. Vemuri, Nonlinear dynamics of a 695 semiconductor laser with filtered optical feedback and the in-

11 fluence of noise, Phys. Rev. E 67 (2003) 046213. doi:10.1103/ PhysRevE.67.046213

URL http://link. aps .org/doi/10.1103/PhysRevE.67.046213

[22] R. L. Honeycutt, Stochastic runge-kutta algorithms. i. white

700 noise Phys. Rev. A 45 (1992) 600-603. doi:10.1103/PhysRevA. 45.600

URL http://link.aps.org/doi/10.1103/PhysRevA.45.600

[23] C. Masoller, Noise-induced resonance in delayed feedback systems Phys. Rev. Lett. 88 (2002) 034102. doi:10.1103/PhysRevLett.88.034102

705 URL http://link.aps.org/doi/10.1103/PhysRevLett.88. 034102

[24] A. Hohl, A. Gavrielides, Bifurcation cascade in a semiconductor laser subject to optical feedback Phys. Rev. Lett. 82 (1999)

710 1148-1151. doi:10.1103/PhysRevLett.82.1148

(1) URL http://link.aps.org/doi/10.1103/PhysRevLett.82. 1148

[25] A. Fischer, M. Yousefi, D. Lenstra, M. Carter, G. Vemuri, Experimental and theoretical study of semiconductor laser dynamics due to filtered optical feedback, Selected Topics in Quantum Electronics, IEEE Journal of 10 (5) (2004) 944-954. doi:10.1109/JSTQE. 2004.835997

[26] H. Erzgräber, B. Krauskopf, Dynamics of a filtered-feedback laser: influence of the filter width Opt. Lett. 32 (16) (2007) 2441-2443. doi:10.1364/OL.32.002441

URL http://ol . osa. org/abstract . cfm?URI=ol-32-16-2441

[27] T. Erneux, G. Hek, M. Yousefi, D. Lenstra, The injection laser limit of lasers subject to optical feedback (2004). doi:10.1117/ 12.545898

URL http://dx.doi.org/10.1117/12.545898 